\title{
Life Substance and Life Materials
}

\author{
Yan Jinzhong ${ }^{1}$ and Yan Zhangxi ${ }^{2}$ \\ 1. Changsha Jiageer Machinery Manufacturing Co., Ltd., Pingtang Town, Changsha City 410208, Hunan, China \\ 2. Beijing Didi Infinite Technology Development Co., Ltd., Beijing 100086, China
}

\begin{abstract}
Life material is the result of the unification of physics and biology. The life material refers to material that has four-season structure, can automatically carry out the four-season process, automatically respond to the internal and external environments, and has life vitality. Its greatest feature is the presence of substances with opposite properties and neutral substances within it. These substances with opposite properties and functions interconnect, mutually constrain, and compete with each other to form an automatic substance flow, energy flow, and information flow within the material. All substances in the universe are living substances. The life characteristic of the substance is its four-season characteristics, that is, the various properties of the substance. Living materials include organic life materials and inorganic life materials, including natural living materials and artificial living materials. Protein is an organic life material or substance composed of 5 categories and 20 kinds of amino acids. Smart materials and intelligent materials have incomplete four-season structure and are quasi-inorganic life materials. Soil is the natural inorganic living material. The basic characteristics of artificial inorganic living materials were analyzed with reference to proteins and soils.
\end{abstract}

Keywords: Life material, life substance, protein, soil, inorganic life material.

\section{Introduction}

In the literature [1], the author proposed the concept of life material, and pointed out that protein is organic life material (substance), while intelligent material is quasi-inorganic living material with incomplete four-season structure. They are able to automatically conduct transverse four-season movement, but not Longitudinal four-season transformation.

The life material is a major category of materials, including natural living materials and artificial living materials. Protein is an organic life material or substance, including natural and synthetic proteins [1]. Corresponding to proteins in living things, natural inorganic living materials must exist on the earth.

Materials are the foundation of the artificial world and play an important role in modern society. The development of materials has experienced the stages of natural materials, synthetic materials, and composite materials. Now it is in the period of functional materials and smart materials. Intelligent

Corresponding author: Yan Jinzhong, Senior Engineer, research field: disciplinary sciences. E-mail: yanjinzhong32@sina.com. material system has also appeared [3]. After figuring out the structure of living materials and finding out natural inorganic living materials, inorganic living materials can be artificially manufactured.

Inorganic life materials are one of the key technologies of the new technological revolution [4], and its emergence will completely change artificial system and human society. The study of life materials and inorganic life materials has great significance.

\section{Life Materials and Their Characteristics}

The life materials refer to materials that have a four-season structure, can automatically perform the dynamics of the four seasons, and can automatically respond to changes in the internal and external environment. According to this definition, life materials have the following characteristics.

1) It has a four-season structure.

In the living materials, the four-season mechanisms - spring, summer, center, autumn and winter mechanisms exist simultaneously, that is, spring, summer, center, autumn and winter each carry out their own functions, and automatically coordinate with 
each other according to the requirements of the four seasons (see Fig. 1).

2) Characteristics of four-season structure.

The characteristics of the spring mechanism. Spring is vitality. The spring mechanism has meanings of opening, decomposition, promotion and smoothness.

The characteristics of the summer mechanism. Summer is hot. The summer mechanism releases energy.

The characteristics of the autumn mechanism. Autumn is killing and blocking. The autumn mechanism has meanings of closing, synthesis and prevention.

The characteristics of the winter mechanism. Winter is cold. The winter mechanism is concealing and it is about energy storage.

The characteristics of the center mechanism: The center is the integration, the hub and the control.

In the four-season materials, spring and summer are rising and releasing, and autumn and winter are falling and storing, that is, substances, processes and actions with opposite properties exist simultaneously. For example, acid and base, oxidation and reduction, decomposition and synthesis, energy release and energy storage, etc. exist in the four-season materials simultaneously. The living material is overall stable and the interior is dynamic. All mechanisms in the four seasons remain independent and do not mutate or transform. These are the biggest characteristics of living materials. Life materials are also known as four-season materials.

3) The substance composition characteristics of the four-season materials. The energy-releasing substances and the energy-storing substances, the active substances and the stable substances, the neutral substances, that is, the substances with opposite natures, and neutral substances exist in the four-season living materials.

4) Substance flow, energy flow, and information flow in living materials. In the four-season materials, the substances and systems with opposite properties exist at the same time. They interconnect and compete with each other to promote the existence of substance flow, energy flow and information flow in the material, so that the material has living vitality. While the neutral substances and systems maintain the stability and balance of life materials.

5) Due to the four-season confinement [5], life materials have the ability to automatically self-detect and self-repair.

6) The four-season material is a unified whole, and all the mechanisms and substances are closely connected, that is, there are link-up materials in the four- season materials.

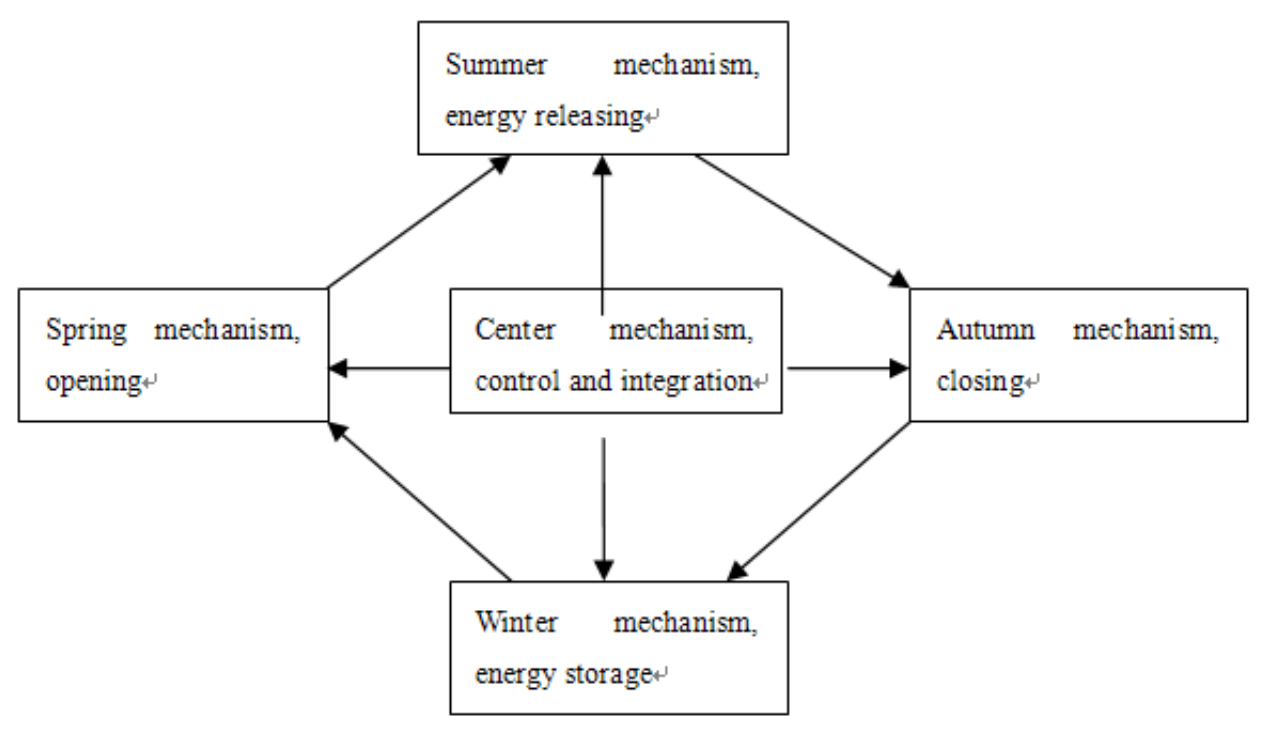


Fig. 1 Four-season structure of life materials.

Living materials include natural living materials and artificial living materials.

\section{The Living Nature and the Four-Season Classification of Substance}

\subsection{The Nature of Substance Is the Nature of Life}

The universe is life, and is made up of different kinds of substances. The structure and functions of life in the universe are realized by various substances. The nature of substance constitutes the nature of life. Life is an automatic mechanism, so the substances that constitutes life are also automatic substances, that is, the substance can automatically move, automatically react and change, automatically generate and develop, conduct automatic cognition and thinking, automatic organization, autonomous behavior and automatic control. The first impulse of the universe [6] is expressed by substances. All substances are life substances.

\subsection{Four-Season Classification of Substances}

Each season of the four-season material consist of the corresponding substances. In this way, according to the nature of the four seasons, various substances can be classified into four seasons.

1) Spring substances. Spring is alive and active, and it is smooth, scattered, becoming light, decomposition, releasing and rising. Spring substances are substances that are easily decomposed or substances that are initially active.

2) Summer substances. Summer is hot. Summer substances are energy substances, the most active substances.

3) Autumn substances. Autumn is killing and blocking; it is stable and restraint, condensation and becoming heavy, sinking and synthesis. Autumn substances are synthetic substances or initially stable substances.

4) Winter substances. Winter is storing. Winter substances are the energy storage substances, the most stable substances.

5) Control substances. In the four seasons, the center is closely connected with the four seasons, and can control the four seasons. It is a combination of four-season substances of spring, summer, autumn and winter, and it contains four-season substances.

In addition to the four-season substances mentioned above, there are various transitional and auxiliary substances whose properties are the betweenness of the four seasons.

\subsection{The Nature of the Substance Is Classified According to Its Strength and Weakness}

1) Strong, weak, and medium types of the same strength nature

Substance natures include strength, motion, vibration, electrical property, magnetic property, chemical activity, taste, odor, biological activity etc. Each property can be divided into strong, weak, medium or active, stable and neutral. This is the manifestation of integrating three into one in Tai Ji [7].

2) Multi-season classification within the same strength nature

From the strongest (most active) to the least active (most stable), the same properties can be divided into $2,5,8,16$ and 32 types of states, which are multi-season classifications of the same strength nature.

3.4 Four-Season or Multi-Season Distribution Diagram of Substances According to Their Strength Nature

1) Four-season distribution diagram of substances strength nature

The distribution of strength and weakness of a certain nature is the four-season distribution. In the four seasons, the winter solstice is the most energy-constrained, and the summer solstice is the 
most energy-spreading. From the winter solstice, through the spring to the summer solstice, the energy is gradually released to the maximum, that is, the activity of the substances is gradually increased. From the summer solstice, through the autumn to the winter solstice, the energy is gradually restrained to the minimum, that is, the stability of the substances is gradually enhanced (see Fig. 2).

2) Four-season distribution of each element periodic activity in the periodic table of elements

The eight elements of the third cycle of the periodic table of elements: $\mathrm{Na}, \mathrm{Mg}, \mathrm{Al}, \mathrm{Si}, \mathrm{P}, \mathrm{S}, \mathrm{Cl}, \mathrm{Ar}$ are distributed in the four seasons figure according to the activity and stability, as shown in Fig. 3.
Fig. 3 shows that the eight elements in the third cycle can be distributed in four seasons according to the activity-stability. Elements of other cycles can be similarly distributed. In the first cycle, there is only $\mathrm{H}$ and $\mathrm{He}$, which are distributed in the summer solstice and winter solstice respectively. This cycle has only two seasons.

The element as a nuclide is a two-dimensional substance [8]. All elements are four-season living substances.

3) Four-season distribution of nature strength of simple substance and compound

Similarly, each nature of simple substance and compound can be classified into active, neutral, and stable,

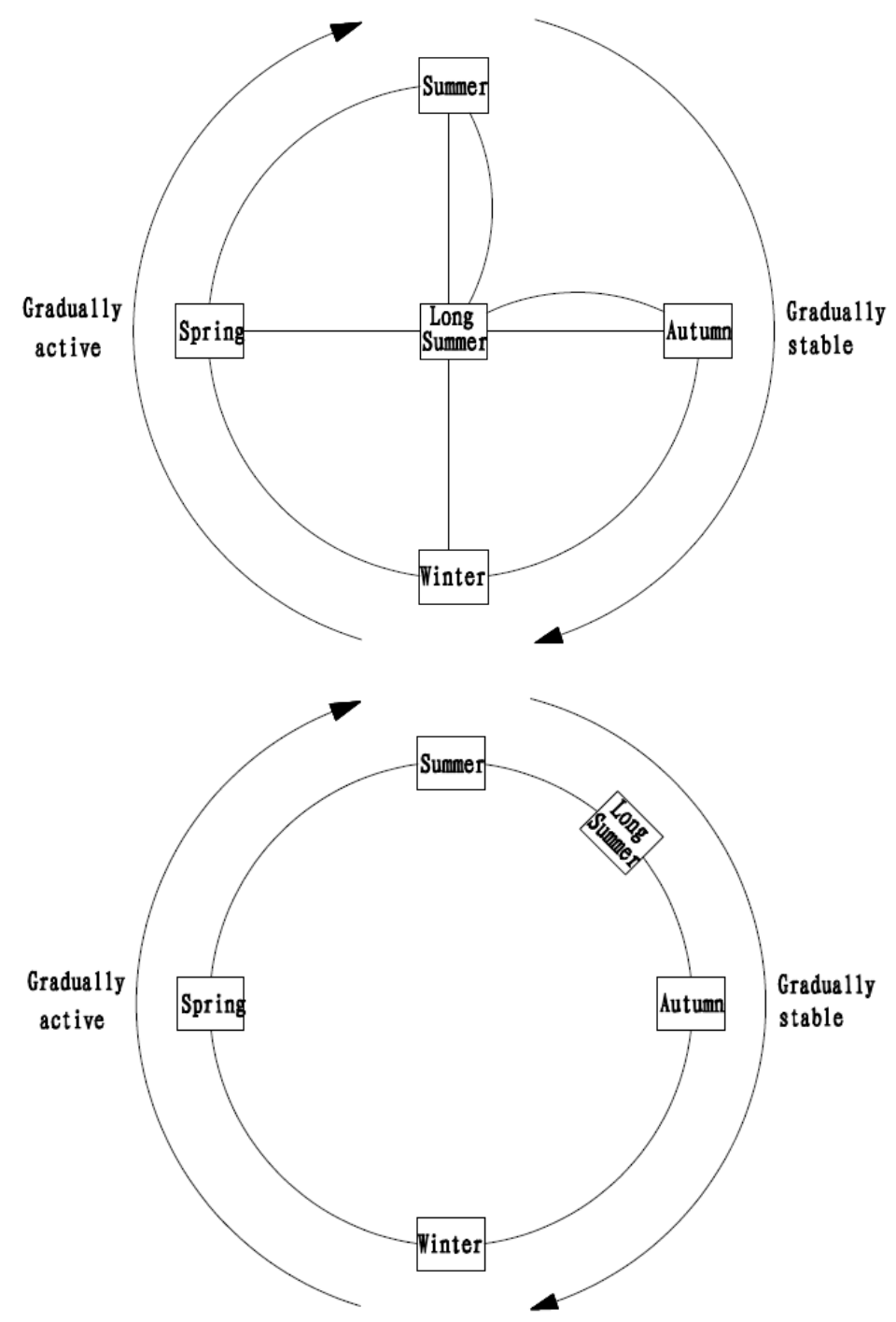

Fig. 2 Changes of up and down in the four seasons of substance nature. 


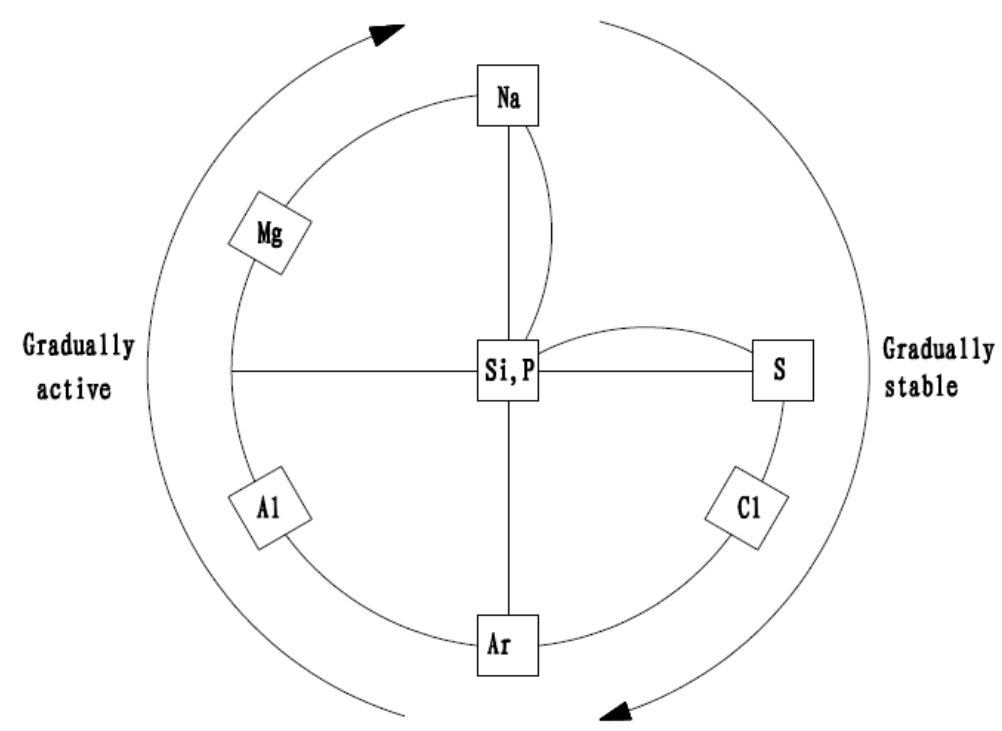

Fig. 3 Four-season distribution of elemental activity-stability in the third cycle.

and can be distributed in four seasons or multiple seasons. They are all four-season life substances. All kinds of substances in living things are naturally life substances.

All substances in the universe are living substances.

\section{Organic Four-Seasons Living Substance - Protein}

Organic living substances are important components of living things - proteins, including natural and synthetic proteins (insulin, etc.).

Protein is the four-season whole with living vitality containing five categories and 20 kinds of amino acids. The five categories of amino acids are: non-polar aliphatic amino acids, polar neutral amino acids, aromatic amino acids, acidic amino acids, and basic amino acids.

In protein, acidic and basic amino acids exist simultaneously; positively charged, negatively charged and uncharged amino acids exist simultaneously; polar, non-polar, and neutral amino acids exist simultaneously; hydrophilic and hydrophobic amino acids exist simultaneously; stable and active amino acids exist simultaneously [9]. This is a typical feature of living materials. Therefore, the protein composed of amino acids is an organic four-season living substance [1].

\section{Natural Inorganic Life Materials - Soil}

In contrast to organic life - living things and inorganic life - the solar system and the earth [1], you can find inorganic life materials in nature. In the five Xings figure of the four seasons, the earth is in the center [7], which is the combination of four seasons, contains four-season substances, and has the structure and functions of four seasons. So the natural inorganic living material is the soil. In Chinese, earth refers to soil.

\subsection{Soil Life Structure}

The soil consists of three types of substances, which are solid, liquid and gas [10].

1) Solid substance is the main body of the soil, including minerals and organic substance etc.

2) Soil liquid mainly refers to moisture, including many dissolved substances.

3) Soil gas is air existing in soil pores, most of which are oxygen, nitrogen and others from the air.

In the soil, the solid is stable mechanism; the liquid is control mechanism, and the gas is change mechanism [1]. These three types of substances constitute the life structure of the soil. 


\subsection{Soil Contains All Substances in the Four Seasons}

Almost all stable and unstable elements in the periodic table (except artificial radioactive elements) can be found in the soil [11], but the contents of various elements in different soils are different. In the soil, substances with opposite nature such as positive and negative charges, acid and base, active and stable substances exist in the soil simultaneously, and neutral substances are also abundant in the soil. It shows that the soil has the four-season structure.

\subsection{Four-Season Response in Soil and Formation of} Substance Flow, Energy Flow, and Information Flow

1) Adsorption and desorption of soil. The soil colloidal particles are small, and have a large specific surface area, and conduct cation adsorption and anion exchange adsorption.

2) Acidity and oxidation-reduction. There are both acidic and basic substances in the soil, and there exists a variety of oxidizing and reducing substances, which make the soil have both oxidizability and reducibility.

3) Positive and negative electric charges exist simultaneously. There are many types of electric charges in the soil, including permanent negative electric charges, variable negative electric charges, and positive electric charges. The soil colloid is an amphoteric colloid with both positive electric charges and negative electric charges.

4) Soil substance transfer. There is aqueous solution in the soil. The soil solution is at the interface between the solid phase and the liquid phase in the three-phase system of the soil, and some chemical processes of the soil, including the conversion and migration of nutrients, are carried out at the interface [12].

\subsection{Soil Colloids Maintain Stability of the Soil Structure and Internal Activity}

Soil colloids are both cohesive and dispersive. Since the specific surface area and surface energy of the colloid are large, in order to reduce the surface energy, the colloid has a tendency to attract and agglomerate each other, which is the cohesiveness of the colloid. However, in the soil solution, the colloid often has negative electric charges, that is, it has negative zeta potential; so the colloidal particles are mutually repelled due to the same electrical properties. The higher the zeta potential, the stronger the repulsive force is and the more dispersed the colloidal particles are. The cohesiveness of soil colloids keeps the structure of the soil stable, and the dispersion of soil colloids keeps the interior of the soil active [12].

The composition and many characteristics of the soil indicate that the soil is the living material which is composed of inorganic materials with a four-season structure and which is capable of automatically performing four seasons. It is the natural inorganic life material and the prototype of artificial inorganic life materials.

\section{Artificial Quasi-Living Materials - Smart Materials, Intelligent Materials}

\subsection{The Two-Season Structure of Smart Materials}

Smart materials can respond automatically to the environment, indicating that it is a living substance. The smart material is reversible material, that is, the material can move and change in the forward direction, and can also move and change in the opposite direction, and the amount of positive and negative motion and change is equal [13] (see Fig. 4).

The smart material has two states: the initial state and the responsive state, which are equivalent to the spring and autumn states in the four seasons. It does not have a complete four-season structure and is the quasi-inorganic living material.

\subsection{Four-Season Structure and Dynamics of Intelligent Materials}

Generally, intelligent materials are composed of four parts: base material, sensitive material, driving material and information processor [3]. It contains three parts: 1) the bearing part, namely, the base material; 2) the information processing part, namely, 


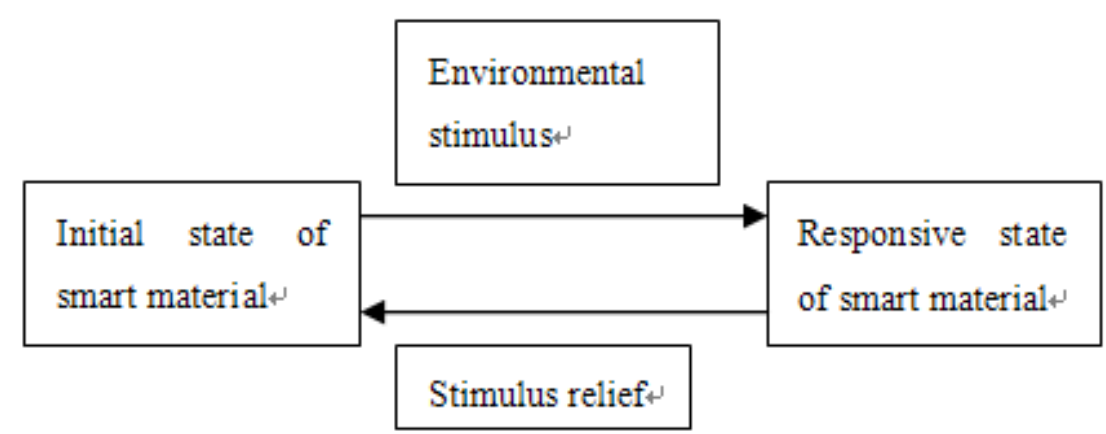

Fig. 4 Dynamics of smart materials.

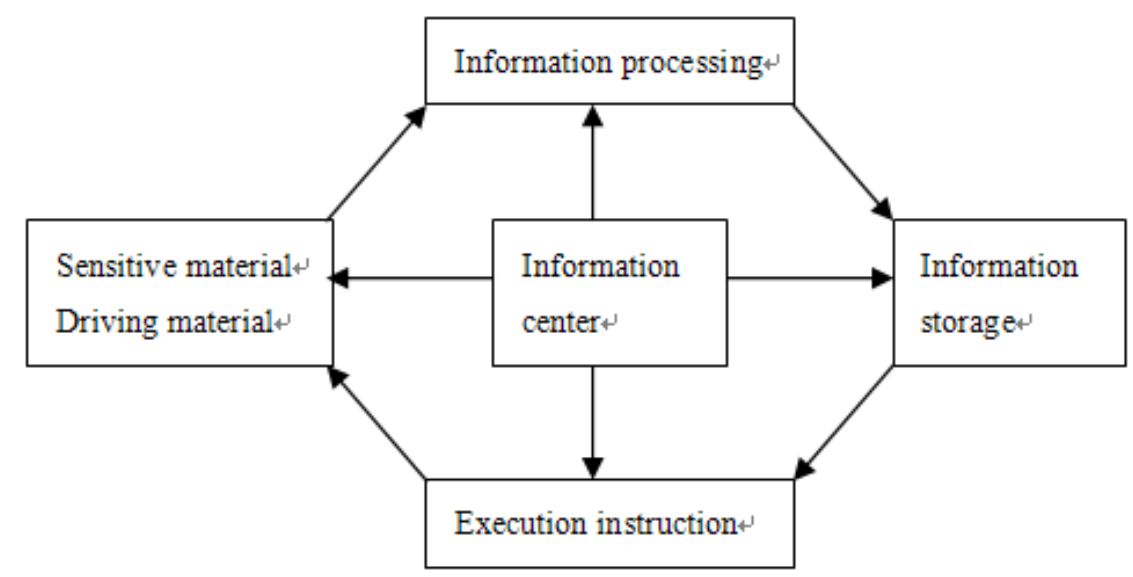

\section{Base material}

Fig. 5 Four-season structure of intelligent materials.

information perception, information processing, information storage, execution instruction sending; and 3) execution part-driving material. Information sensing-sensitive materials and execution mechanism-driving materials are combined into one. The four-season structure of intelligence materials is shown in Fig. 5.

The intelligent material system has a more complete informational four seasons and can be automatically perceived and executed. But it has no material four seasons and energy four seasons, so it is the quasi-inorganic living material.

\section{Structure and Substance Characteristics of Artificial Inorganic Living Materials}

Inorganic living materials are living materials composed of inorganic substances. Referring to the life material and the natural inorganic life material soil, several characteristics of the artificial inorganic living materials can be found.

\subsection{System of Inorganic Life Materials}

The complete inorganic life material consists of five parts: 1) the structure or the bearing part, which should be integrated with other parts; 2) the informational four seasons, including information sensing; 3) the energy four seasons; 4) the material four seasons; 5) the execution system.

These five parts are in the same system and are interlaced.

\subsection{Substance Characteristics of Artificial Inorganic} Living Materials

1) Choice of particle size. A substance with a small 
particle size is active and sensitive, so the living material contains a large amount of small particles. However, substances with a small particle size have poor stability. In order to ensure the stability of the material, the living material should contain enough large particulate substance.

2) Selection of four-season substances. Various substances of opposite nature and neutral substances exist simultaneously in the living material, facilitating the formation of four-season process. At the same time, transitional substances between the seasons are also necessary.

3) Selection of structural substances. The overall structure of the material should be a three-dimensional reticular substance with good stability (mechanical properties) and sensitivity. It can connect and accept all substances, and can integrate with other substances to participate in the four-season process.

\section{Conclusions}

1) Life materials are materials that have four-season structure, are capable of automatically performing four-season dynamics, can actively respond to internal and external environments and have living vitality.

2) Among the life materials, the spring mechanism is opening and decomposition; the summer mechanism is the release of energy; the center mechanism is the hub; the autumn mechanism is closing and synthetic; and the winter mechanism is the storage of energy. Spring and summer are rising and releasing; autumn and winter are the declining and storage, and the center mechanism is balance and coordination. Substances and processes of opposite nature exist in living materials at the same time. The living materials are overall stable; the interior is dynamic, and it automatically performs various four-season processes and responds to internal and external environments. All mechanisms in the four seasons remain independent and do not mutate or transform.

3) The nature of substance is the nature of life, and all substances are life substances. A variety of different substances constitute the four-season distribution. The strength of a certain nature corresponds to different substances, and this also constitutes the distribution of the four seasons.

4) Protein is composed of five (four seasons) amino acids, which are organic living substances (materials).

5) Soil is the natural inorganic life material. It consists of three substances: solid, liquid and gas. It contains substances of opposite nature such as positive and negative electric charges, acids and bases, active and stable substances, and neutral substances. Chemical adsorption, acid-base interaction, oxidation and reduction, etc. in the soil cause automatic four-season reaction and movement in the soil, forming material flow, energy flow and information flow in the soil. The soil colloid keeps the soil structure stable and internally active.

6) The smart material is the quasi-inorganic living material with two states. While the intelligent materials have informational four seasons and actuators, no substance flow and energy flow, and are quasi-inorganic living materials.

7) The complete artificial inorganic living material has five parts, and the selection of substance depends on the type of substance and the particle size.

\section{References}

[1] Jinzhong Yan 2013. "Introduction to Unity of Physics and Biology, Inorganic Life Materials." Journal of Physical Science and Application 3 (1): 58-64.

[2] Yuezeng Chen 2000. General Biology. Beijing: Higher Education Press, pp. 23-27, 62-63.

[3] Xinwo Zhou, 2002. Functional Materials Science. Beijing: Beijing Institute of Technology University Press, pp. 1-10, 391-395.

[4] Jinzhong Yan 2014. "Beginning of A New Revolution of Science \& Technology." Journal of Physical Science and Application 4 (4): 269-275.

[5] Jinzhong Yan 2015. "The Four-Seasonal Movement of Life." Journal of Life Science 9: 131-138.

[6] Jinzhong Yan 2014. "The First Push of the Universe and Origin of Life." Journal of Physical Science and Application 4 (3): 198-205. 
[7] Jinzhong Yan 2017. "Disclosing of Thousand Years' Mystery - Origin of the Book of Changes." Journal of Physical Science and Application 1: 8-20.

[8] Jinzhong Yan 2013. "State Space-Time and Four States of Universe." Journal of Physical Science and Application 3 (2): 125-133.

[9] Chenglu Zou 1997. Second Genetic Code. Changsha: Hunan Science and Technology Press, pp. 24-46.

[10] Xueyuan Li 2001. Soil Chemistry. Beijing: Higher Education Press, pp. 100-309.
[11] Daogong Chen 1994. Geochemistry. Hefei: China University of Science and Technology Press, pp. 37-74.

[12] Soil Chemistry, 2018. https://baike.baidu.com /item/\%E5\%9C\%9F\%E5\%A3\%A4\%E5\%8C\%96\%E5\% AD\%A6/8028280.

[13] Kangde Yao, and Guoxiang Cheng 2002. Intelligent Material. Beijing: Chemical Industry Press, pp. 1-2. 